\title{
Sonography of the Accessory Head of the Biceps Brachii
}

Ricardo Augusto Lutterbach-Penna, MD, Monica Kalume Brigido, MD, Brian Robertson, AS, RDMS, Sung Moon Kim, MD, Jon A. Jacobson, MD, David P. Fessell, MD

Received November 26, 2013, from the Division of Musculoskeletal Radiology, University of Michigan Health System, Ann Arbor, Michigan USA. Revision requested December 14, 2013. Revised manuscript accepted for publication January 30, 2014.

Address correspondence to David P. Fessell, MD, Division of Musculoskeletal Radiology, University of Michigan Health System, 1500 E Medical Center Dr, SPC 5030, Ann Arbor, MI 48108 USA.

E-mail:fessell@umich.edu

Abbreviations

MRI, magnetic resonance imaging

doi:10.7863/ultra.33.10.1851
Anatomic variations in the anterior aspect of the shoulder, such as an accessory head of the biceps brachii muscle, are not uncommon. The magnetic resonance imaging and arthroscopic appearance of the accessory head of the biceps brachii has been recently described. This series demonstrates the sonographic appearance of the accessory head of the biceps brachii in the bicipital groove. It is an asymptomatic, flat, echogenic structure with average measurements of $7.7 \times 1.2 \mathrm{~mm}$ in cross section. Knowledge of this anatomic variant can avoid the misdiagnosis of a longitudinal split tear and improve the accuracy of sonography.

Key Words - biceps; musculoskeletal ultrasound; shoulder; sonography; tendon; variant
$\Lambda$ natomic variations in the anterior aspect of the shoulder are not uncommon, with a frequency ranging from $2 \%$ to $18 \%$ depending on the ethnic group. ${ }^{1-7}$ Examples include an accessory head of the biceps brachii muscle, coracobrachialis brevis muscle, accessory subscapularis muscle, and variants of the pectoralis muscles. ${ }^{1}$ Correct identification of normal variants is important to avoid misdiagnosis of disorders and for accurate diagnosis. ${ }^{1,4,8,9}$

Sonography has been shown to be an excellent imaging modality to evaluate muscles and tendons, with advantages and disadvantages compared to magnetic resonance imaging (MRI). ${ }^{10,11}$ Recently the MRI and arthroscopic appearance of the accessory head of the biceps brachii has been described. ${ }^{1,9,12-14}$ The purpose of this series is to demonstrate the sonographic appearance of the accessory head of the biceps brachii in the bicipital groove. To our knowledge, no prior ultrasound articles describing this anatomic variant have been published. Knowledge of this anatomic variation and its normal appearance on sonography can help prevent falsepositive diagnoses of proximal biceps disorders. $8,9,15$ 


\section{Materials and Methods}

Institutional Review Board authorization was obtained for this retrospective study, and informed consent was waived. Four cases of an accessory head of the biceps brachii with an otherwise normal long head of the biceps brachii tendon were identified from a database search and from our clinical experience between May 17, 2010, and January 4, 2012. The sonograms were obtained with a high-frequency linear transducer (LOGIQ 9, 6-15- or 7-11-MHz transducer; GE Healthcare, Milwaukee, WI; or iU22, 12-5- or 17-5-MHz transducer; Philips Healthcare, Bothell, WA). The accessory head of the biceps brachii was identified as a hyperechoic fibrillar structure located superficial and parallel to the long head of the biceps brachii tendon at the level of the bicipital groove (Figures 1 and 2). Measurements of its short and long axes in the transverse (axial) plane were made at the level of the bicipital groove, as previously described. ${ }^{1}$

Patient records were reviewed to assess for MRI of the same shoulder. The sonograms and MR images were reviewed by consensus of 2 musculoskeletal radiologists, each with more than 10 years of experience in musculoskeletal sonography and MRI. The presence of an accessory biceps brachii tendon on MRI was confirmed if a low-signal tendonlike structure was identified parallel and adjacent to the long head of the biceps brachii tendon in the bicipital groove, which originates proximally in the region of the joint capsule, as described in previous studies. ${ }^{1}$ Measurements in the short and long axes in the transverse (axial) plane were also made at the level of the bicipital groove for patients with MRI studies, as previously described. ${ }^{1}$

\section{Results}

The study group consisted of 4 women with an average age of 52 years (range, 31-68 years). The right shoulder was involved in $75 \%$ (3 of 4) and the left in 25\% (1 of 4). Sonographic examinations were performed for a history of shoulder pain. One patient had shoulder MRI 5.9 months after sonography, and a second patient had a shoulder MR arthrogram 3.7 months after sonography. Both MRI studies confirmed the accessory biceps tendon.

Sonographic measurements of the accessory head of the biceps brachii in the short and long axes in the transverse (axial) plane showed an average anteroposterior dimension of $7.7 \mathrm{~mm}$ (range, $6.2-10.0 \mathrm{~mm}$; SD, $1.6 \mathrm{~mm}$ ). The short-axis measurement had an average mediolateral dimension of $1.2 \mathrm{~mm}$ (range, 0.7-1.9; SD, $0.5 \mathrm{~mm}$ ). The craniocaudal dimension could not be reliably measured on these retrospective images.
For the 2 patients who had both sonography and MRI, the accessory biceps measured $1.5 \times 6.9 \mathrm{~mm}$ on sonography and $1.6 \times 7.2 \mathrm{~mm}$ on MRI in the first patient and $1.2 \times 6.5$ mm on sonography and $1.4 \times 6.3 \mathrm{~mm}$ on MRI in the second patient (anteroposterior $\times$ mediolateral dimensions).

\section{Discussion}

In the adult, the biceps brachii is a large fusiform muscle in the flexor compartment of the upper arm, with the long head arising from the supraglenoid tubercle and the short head arising from the coracoid process. ${ }^{2,3,5,16-18}$ Both heads descend along the anterior aspect of the upper arm to insert into the radial tuberosity. $2,3,5,16$

Biceps brachii muscle is variable in morphologic characteristics and the number of heads, ranging from 3 to 7.,18,19 The accessory tendon(s) can arise from the coracoid

Figure 1. A, Transverse sonogram showing the accessory head of the biceps brachii, identified as a hyperechoic fibrillar structure (arrow) located superficial and parallel to the long head of the biceps tendon (asterisk) in the bicipital groove. B, Corresponding axial T1-weighted fat-saturated MR arthrogram confirming this finding (arrows). BT indicates biceps tendon; GT, greater tuberosity; L, lateral; and M, medial.

A

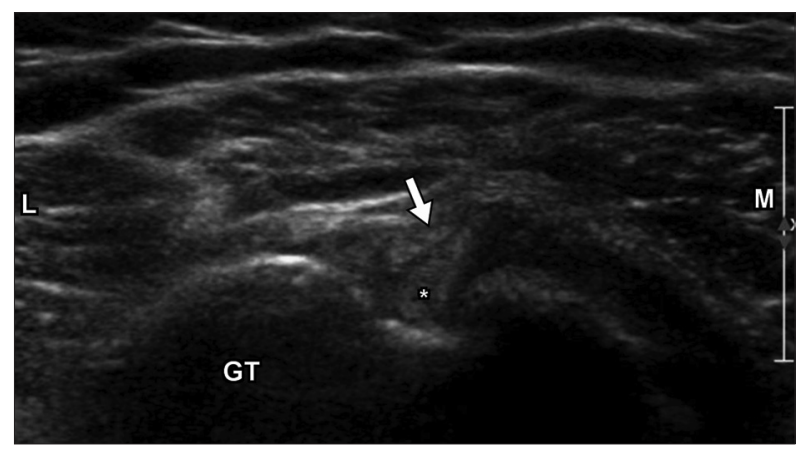

B

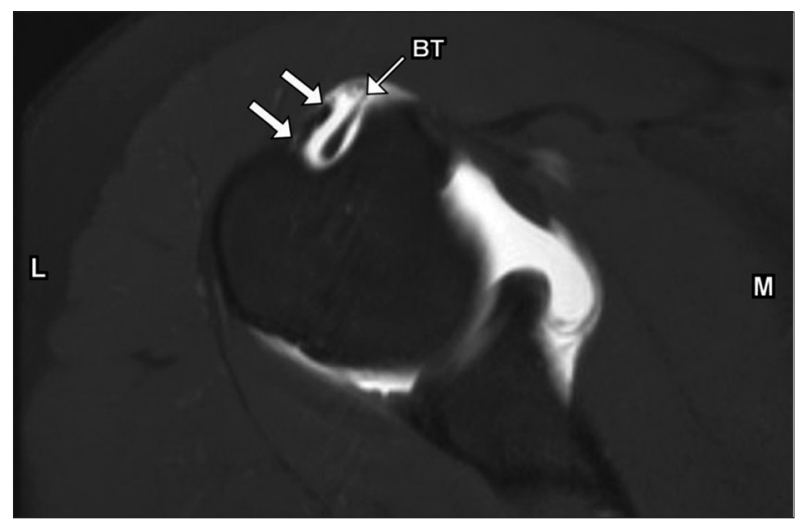


process, pectoralis minor tendon, proximal head of the humerus, articular capsule of the humerus, or anterior surface of the distal part of the pectoralis major. ${ }^{20,21}$ The most common variation arises from the proximal humerus, known as the humeral head or the third head of the biceps brachii. $^{21}$

Magnetic resonance imaging and sonography are the two most commonly used imaging techniques for evaluation of tendon disorders. ${ }^{10,11}$ A recent cadaveric study with MRI correlation showed the accessory head of biceps brachii muscle at the anterolateral aspect of the biceps brachii long-head tendon, at the level of the bicipital groove. ${ }^{1}$ Arthroscopic reports have confirmed an accessory tendon at this site, consistent with a normal variant. ${ }^{12-14}$ In the study by Gheno et al, ${ }^{1}$ the average short- and longaxis dimensions of the accessory biceps were 0.85 and $6.2 \mathrm{~mm}$ at the level of the bicipital groove. In our series, we found an average short-axis maximum dimension of $1.2 \mathrm{~mm}$ and long-axis maximum dimension of $7.7 \mathrm{~mm}$. This difference may be due to the fact that the previous cadaveric study included only 2 specimens with an accessory biceps brachii.

The primary differential diagnosis for a linear echogenic structure within the bicipital groove is a longitudinal split of the long head of the biceps tendon. ${ }^{1,4,8,9}$ An asymptomatic flat echogenic structure immediately adjacent to the anterolateral aspect of an otherwise normal biceps tendon at the level of the bicipital groove, with an origin as discussed above and insertion at the medial side of the common biceps tendon, is consistent with a normalvariant accessory biceps. ${ }^{1,8,9,16,20-22}$ This normal variation should not be mistaken for a tear or longitudinal split of the proximal long head of the biceps brachii. ${ }^{8,9}$ Sonography

Figure 2. Transverse sonogram showing the accessory head of the biceps brachii from another patient, identified as a hyperechoic fibrillar structure (arrows) located superficial and parallel to the long head of the biceps tendon (asterisk) in the bicipital groove. Abbreviations are as in Figure 1.

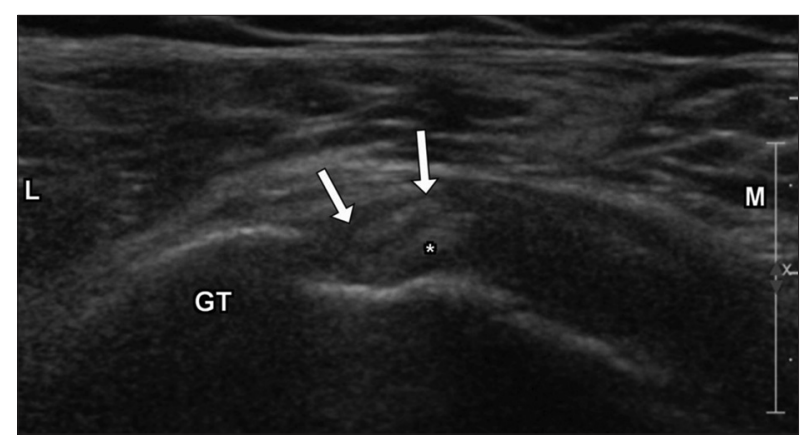

allows the sonographer to assess for pain at the proximal biceps brachii and allows the use of gentle transducer pressure to elicit symptoms. ${ }^{11}$ This factor provides an advantage compared to MRI and can also be helpful in distinguishing a normal variant from a split biceps brachii tendon.

The accessory biceps tendon was visualized in the transverse plane, separate from and just superior to the level of the subscapularis tendon fibers. Fluid within the biceps tendon sheath may aid imaging of an accessory tendon; however, this aspect requires further study. In our limited experience, the accessory biceps is much better visualized in the transverse plane compared to the longitudinal plane; however, a prospective study would better assess this point.

It is unclear whether the accessory tendon of the biceps brachii could be the source of shoulder symptoms. ${ }^{8,14}$ Dierickx et al ${ }^{13,15}$ speculated that the accessory biceps may cause impingement in a young patient, which could evolve into a rotator cuff tear. Others described the accessory tendon as a benign anatomic variant that does not contribute to shoulder disorders. ${ }^{12}$ More study is needed to determine whether this normal variant is ever symptomatic or pathologic or whether it can predispose to biceps tenosynovitis or other disorders.

This study had several limitations. It was a retrospective series with a small sample size and lacked surgical correlation. However, this entity has been described as a normal variant, and such patients would not be expected to go to surgery. This variant has been previously described on MRI and arthroscopy, and MRI was performed in 2 of our 4 cases, confirming an accessory head of the biceps brachii. All 4 cases were female patients. Per our literature review, this anatomic variant has not been noted to have a female predominance. This finding may have been due to the small number of cases.

Knowledge of the accessory head of the biceps brachii and its appearance on sonography is important to avoid misdiagnosis of disorders. The accessory head of the biceps brachii has a characteristic appearance on sonography and should not be mistaken for a longitudinal split of the proximal aspect of the long head of the biceps brachii tendon.

\section{References}

1. Gheno R, Zoner CS, Buck FM, et al. Accessory head of biceps brachii muscle: anatomy, histology, and MRI in cadavers. AJR Am J Roentgenol 2010; 194:W80-W83.

2. Rodríguez-VázquezJF, Mérida-Velasco JR, Jiménez-Collado J.Unusual variation of a third head of the biceps brachii muscle. Ann Anat 1999; 181:573-575. 
3. Vollala VR, Nagabhooshana S, Bhat SM, Potu BK, Rakesh V. Multiple accessory structures in the upper limb of a single cadaver. Singapore Med J 2008; 49:e254-e258

4. Rai R, Ranade AV, Prabhu LV, Pai MM, Prakash. Third head of biceps brachii in an Indian population. Singapore Med J 2007; 48:929-931.

5. Asvat R, Candler P, Sarmiento EE. High incidence of the third head of biceps brachii in South African populations. J Anat 1993; 182:101-104.

6. Neto H, Camilli JA, Andrade JCT, Filho JM, Marques MJ. On the incidence of the biceps brachii third head in Brazilian whites and blacks. Ann Anat 1998; 180:69-71.

7. Kopuz C, Sancak B, Ozbenli S. On the incidence of third head of biceps brachii in Turkish neonates and adults. Kaibogaku Zasshi 1999; 74:301305 .

8. Kim KC, Rhee KJ, Shin HD, Kim YM. Biceps long head tendon revisited: a case report of split tendon arising from single origin. Arch Orthop Trauma Surg 2008; 128:495-498.

9. Vinson EN, Wittstein J, Garrigues GE, Taylor DC.MRI of selected abnormalities at the anterior superior aspect of the shoulder: potential pitfalls and subtle diagnoses. AJR Am J Roentgenol 2012; 199:534-545.

10. Lutterbach-Penna RA, Kalume-Brigido M, Robertson BL, Jacobson JA, Girish G, Fessell DP. Deep vein thrombosis simulating hamstring injury on sonography. JUltrasound Med 2012; 31:660-662.

11. Nazarian LN. The top 10 reasons musculoskeletal sonography is an important complementary or alternative technique to MRI. AJR Am J Roentgenol 2008; 190:1621-1626.

12. Wittstein J, Lassiter T, Taylor D. Aberrant origin of the long head of the biceps: a case series. J Shoulder Elbow Surg 2012; 21:356-360.

13. Dierickx C, Ceccarelli E, Conti M, Vanlommel J, Castagna A. Regarding "Aberrant origin of the long head of the biceps: a case series." J Shoulder Elbow Surg 2012; 21:e20-e22.

14. EnadJG. Bifurcate origin of the long head of the biceps tendon. Arthroscopy 2004; 20:1081-1083.

15. Dierickx C, Ceccarelli E, Conti M, Vanlommel J, Castagna A. Variations of the intra-articular portion of the long head of the biceps tendon: a classification of embryologically explained variations. J Shoulder Elbow Surg 2009; 18:556-565

16. Warner JJ, Paletta GA, Warren RF. Accessory head of the biceps brachii: case report demonstrating clinical relevance. Clin Orthop Relat Res 1992; 280:179-181.

17. SwieterMG, CarmichaelSW. Bilateral three-headed biceps brachii muscles. Anat Anz 1980; 148:346-349.

18. Rodríguez-Niedenführ M, Vázquez T, Choi D, Parkin I, Sañudo JR. Supernumerary humeral heads of the biceps brachii muscle revisited. Clin Anat 2003; 16:197-203.

19. Abu-Hijleh MF. Three-headed biceps brachii muscle associated with duplicated musculocutaneous nerve. Clin Anat 2005; 18:376-379.

20. Sargon MF, Tuncali D, Celik HH. An unusual origin for the accessory head of biceps brachii muscle. Clin Anat 1996; 9:160-162.

21. Kumar H, Das S, Rath G. An anatomical insight into the third head of biceps brachii muscle. Bratisl Lek Listy 2008; 109:76-78.
22. Nakatani T, Tanaka S, Mizukami S. Bilateral four-headed biceps brachii muscles: the median nerve and brachial artery passing through a tunnel formed by a muscle slip from the accessory head. Clin Anat 1998; 11:209212. 